All submitting authors are asked to announce the first and last author's potential conflict of interest regarding their abstract.

The following authors have declared relationships:

\title{
Relationships with
}

Al-Batran, S.

Attali, $P$.

Bargou, $R$.

Bauer, $S$.

Beck-Mannagetta, J.

Beelen, D. W.

Behre, G.

Bergner, $R$.

Bethge, W. A.

Bogen, A.

Bokemeyer, $C$.

Brück, $P$.

Brüggemann, $M$.

Brünnert, $D$.

Bumeder, I.

Buske, C.

Butscheid, $Y$.

Cerny, D.

Cerny, $T$.

Christ, $O$.

Cornely, O. A.

Deak, E.

del Giglio, A.

Deschler, $B$.

Doehn, C.

Dreyling, $M$.

Eberhardt, W. E. E.

Egerer, $G$.

Eggermont, A. M.

Eichhorst, B. F.

Elmaagacli, A. H.

Elter, $T$.

Engert, $A$.

Folprecht, G.

Frank, $O$.

Freier, $W$.

Fruehauf, $S$.

Galle, $P$.

Gastl, G.

Geiger, G.
Sanofi-Aventis

BioAlliance Pharma, Paris

Johnson \& Johnson, Micromet AG

Novartis

GABA Internat., Münchenstein, Schweiz

Fa. Medac GmbH

FA Genzyme

Roche Pharma

Bayer-Vital

Wilhelm-Sander-Stiftung, München

Bayer AG

Novartis-Stiftung

Wilhelm Sander-Stiftung

Leukämie Liga e. V.

Deutsche José Carreras Leukämie-Stiftung

Roche

Novartis Pharma GmbH

Pfizer Switzerland

Pfizer Switzerland

Wilhelm-Sander-Stiftung, München

Astellas, Basilea, F2G, Gilead, Pfizer, Merck, Mölnlycke, Nektar, Schering-Plough,Zeneus; German Federal Ministry of Research and Education, Genzyme, SpePharm, United Medical, Vicuron

BMBF Deutschland

BioGeneriX AG

José Carreras Leukämie Stiftung

Bayer Healthcare AG, LioNova, Pfizer, Wyeth

Lilly, Jansen Cilag Mundipharma

Bayer-Schering-Pharma AG

MSD SHARP \& DOHME GMBH

Shering Plough

Relationships exist, but not declared by author

Fa. Novartis

BayerSchering Pharma

BayerSchering Pharma

Merck, Pfizer, Sanofi-Aventis

Stiftung Leukämie e. V., Mannheim

Lilly Deutschland

Paracelsus-Klinik Osnabrück, PAROS Osnabrück,

Genzyme, Novartis

Bayer Healthcare, Bayer Schering Pharma

Novartis

PHYSIOMED ELEKTROMEDIZIN 
Goldschmidt, $H$.

Greten, $T$.

Gromke, $T$.

Habertheuer, K. H.

Haferlach, $T$.

Hahn-Ast, C.

Hehlmann, $R$.

Heidenreich, A.

Heider, U.

Heinemann, $v$.

Heinz, W. J.

Henrich, $D$.

Henschler, $R$.

Hiddemann, $W$.

Ho, A. D.

Hochhaus, A.

Höchsmann, $B$.

Holler, E.

Junghan $B, C$.

Keilholz, U.

Kern, W.

Kiewe, $P$.

Klaskova, K.

Klein, U.

Korfel, A.

Kornacker, $M$.

Kosch, $M$.

Kozak, $T$.

Krause, S. W.

Krekeler, G.

Kuchenbecker, $U$.

Kunzmann, $V$.

Kurth, A. A:

Lange, $T$.

le Coutre, Ph.

Leithäuser, $M$.

Lentzen, $H$.

Leyvraz, $S$.

Linkesch, $W$.

Llovet, J. M.

Lopez, C.

Lorenzen, $S$.

Markova, J.

Mergenthaler, $U$.

Meyer, R. G.

Möhle, $R$.

Montemurro, $M$.

Nagova, L.

Neumann, $F$.
Chugai, Binding Site; Celgene

Bayer Healthcare, Bayer Schering Pharma

Fa. Medac GmbH

Celgene $\mathrm{GmbH}$

MLL Münchner Leukämielabor GmbH, ROCHE Diagnostics, ELN

Ortho Biotech-Division of Janssen Cilag

Deutsche Krebshilfe; Novartis; German José-Carreras-Leukämie-

Stiftung e.V., Competence Network "Akute und chronische

Leukämien", Bundesministerium für Bildung und Forschung,

Germany

Amgen, Bayer, Ferring, Centocor, Wyeth

Novartis

Roche Pharma, Merck Pharma, Sanofi-Aventis; Lilly Deutschland;

Pierre Fabre

Gilead, Schering-Plough, MSD

Roche Pharma

BMBF Deutschland

Roche

Genzyme

Novartis Pharma GmbH

Institute for Clinical Transfusion Medicine and Immunogenetics

Ulm, German Red Cross Blood Donor Service Baden-

Württemberg-Hessen and Institute of Transfusion Medicine,

University of Ulm, Germany

Fresenius AG, Therakos; Deutsche Krebshilfe,

Wilhelm Sander Stiftung

Amgen, Essex Pharma

Novartis

MLL Münchner Leukämielabor $\mathrm{GmbH}$, MHP Münchner

Hämatologiepraxis; Beckman Coulter $\mathrm{GmbH}$

Bayer Schering

Grant MZ CR IGA NR 8033 - 6/2004

Celgene

Bayer Schering

Johnson \& Johnson

Wyeth Pharma GmbH

Grant MZ CR IGA NR 8033-6/2004

Relationships exist, but not declared by author

Wyeth Pharma GmbH

Wyeth Pharma GmbH

Fa. Novartis, Roche

Roche Pharma

Novartis, BMS

Novartis, BMS

Essex Pharma

CYTAVIS BioPharma GmbH

Novartis

Celgene $\mathrm{GmbH}$

Bayer Healthcare, Bayer Schering Pharma

NIH grant

Firma Merck KG

Grant MZ CR IGA NR 8033-6/2004

Novartis, megapharm

Bayer Vital AG

BMBF/Bioprofile

Novartis

Novartis

Leukämie Liga 
Neuwelt, $E$.

Niederwieser, $D$.

Nückel, $H$.

Oettle, $H$.

Ottmann, $O$.

Otto, $T$.

Pardo, L. A.

Patzbecker, U.

Pelz, H.

Petzer, A. L.

Pfreundschuh, $M$.

Platzbecker, U.

Radsak, M. P.

Rebmann, $V$.

Reiter, $A$.

Rick, $O$.

Riess, $H$.

Rohde, D.

Röth, A.

Rugo, $H$.

Rüping, M. J. G. T.

Sayer, H. G.

Schild, $H$.

Schmah, $O$.

Schmitz, S.

Schrezenmeier, $H$.

Schubert, J.

Sezer, 0 .

Siemer, $S$.

Siffert, $W$.

Soelch, J.-P.

Staib, $P$.

Steiner, Th.

Steinmetz, $T$.

Stilgenbauer, $S$.

Tauer, $R$.

Theurich, $S$.

Thiede, $C$.

Thiruchittampalam, D.

Thomas, $L$.

Thomas, R. K.

Topaly, J.

Warmuth, $M$.

Weide, $R$.

Wendtner, C.-M.

Westfeld, $M$.

Willenbacher, $W$.

Wirth, $M$.

Wolf, $J$.

Wulf, G.

Zenz, $T$.
NIH grants no. NS34608, NS33618, and NS44687

Novartis, BMS

Deutsche José Carreras Leukämie Stiftung

sanofi-aventis $\mathrm{GmbH}$; Medac $\mathrm{GmbH}$

BMS, Novartis

Relationships exist, but not declared by author

iOnGen AG.

Pharmion

Wyeth Pharma GmbH

Novartis; Unrestricted Research Grant

Roche, Amgen

Novartis

Deutsche Forschungsgemeinschaft

Deutsche José Carreras Leukämie Stiftung

Novartis Pharma, Germany

Oliver Rick, PHYSIOMED ELEKTROMEDIZIN

Sanofi-Aventis

Bayer Vital GmbH

Geron Corporation, Menlo Park, CA, USA; Alexion Pharma

BMS München

MJGTR, Schering-Plough/Essex, Gilead.

Amgen $\mathrm{GmbH}$, Ortho-Biotec, Novartis $\mathrm{GmbH}$

Deutsche Forschungsgemeinschaft

Telltargeting GbR

Amgen

Institute for Clinical Transfusion Medicine and Immunogenetics

Ulm, German Red Cross Blood Donor Service Baden-

Württemberg-Hessen and Institute of Transfusion Medicine,

University of Ulm, Germany

Roche

Novartis

Bayer AG, Pfizer, Intuitive Surgical System, Bayer Vital GmbH

Eurofins Medigenomix $\mathrm{GmbH}$

Amgen $\mathrm{GmbH}$

TumorTec GmbH

Pfizer Pharma $\mathrm{GmbH}$, Bayer Vital $\mathrm{GmbH}$

Amgen, Ortho Biotec, Roche

Bayer-Schering, Celgene, Roche, GSK

Amgen $\mathrm{GmbH}$

FA Genzyme

AgenDix $\mathrm{GmbH}$

TumoTec $\mathrm{GmbH}$

Roche

Astra Zeneca

Novartis

Novartis

Novartis, megapharm

Relationships exist, but not declared by author

Wyeth Pharma GmbH

Janssen-Cilag; Austrian Myeloma Registry

Bayer Healthcare AG

Novartis

Novartis

Bayer-Schering, Celgene 